Supporting Information

\title{
First Hybrid Embedding Scheme for Polar Covalent Materials Using an Extended Border Region to Minimize Boundary Effects on the Quantum Region
}

\author{
Alexei M. Shor, Elena A. Ivanova Shor, Vladimir A. Nasluzov, \\ Georgi N. Vayssilov, Notker Rösch
}

Table S1. Optimized Gaussian-type orbital exponents (au) the of border centers O*.

\begin{tabular}{rcc}
\hline $\mathrm{s}$ & $\mathrm{P}$ & $\mathrm{d}$ \\
\hline 42.9945813397 & 44.1800166163 & 1.5876992947 \\
15.4994950057 & 10.0730313065 & \\
6.2258536472 & 2.65650061594 & \\
1.0239161449 & 0.73353152694 & \\
0.3209359861 & 0.17372185997 & \\
\hline
\end{tabular}

Table S2. Optimized pseudopotential parameters ${ }^{a}$ (au) of the border centers $\mathrm{O}^{*}$ and $\mathrm{Si}^{\mathrm{PP}}$.

\begin{tabular}{ccccc}
\hline & $l$ & $k$ & $\alpha_{k l}$ & $\mathrm{~A}_{k l}$ \\
\hline $\mathrm{O}^{*}$ & 0 & 1 & 26.023166 & 78.753643 \\
& 0 & 2 & 14.897082 & 15.444531 \\
& 1 & 1 & 23.555342 & -15.348110 \\
& 1 & 2 & 13.256028 & 3.112648 \\
& 2 & 1 & 0.027616 & -0.001791 \\
$\mathrm{Si}^{\mathrm{PP} *}$ & 0 & 1 & 1.756846 & 6.581120 \\
& 1 & 1 & 0.882640 & 2.048685 \\
& 2 & 1 & 0.943234 & -2.036544 \\
\hline
\end{tabular}

${ }^{a}$ For the analytical form of the pseudopotential, see Eq. (1). 

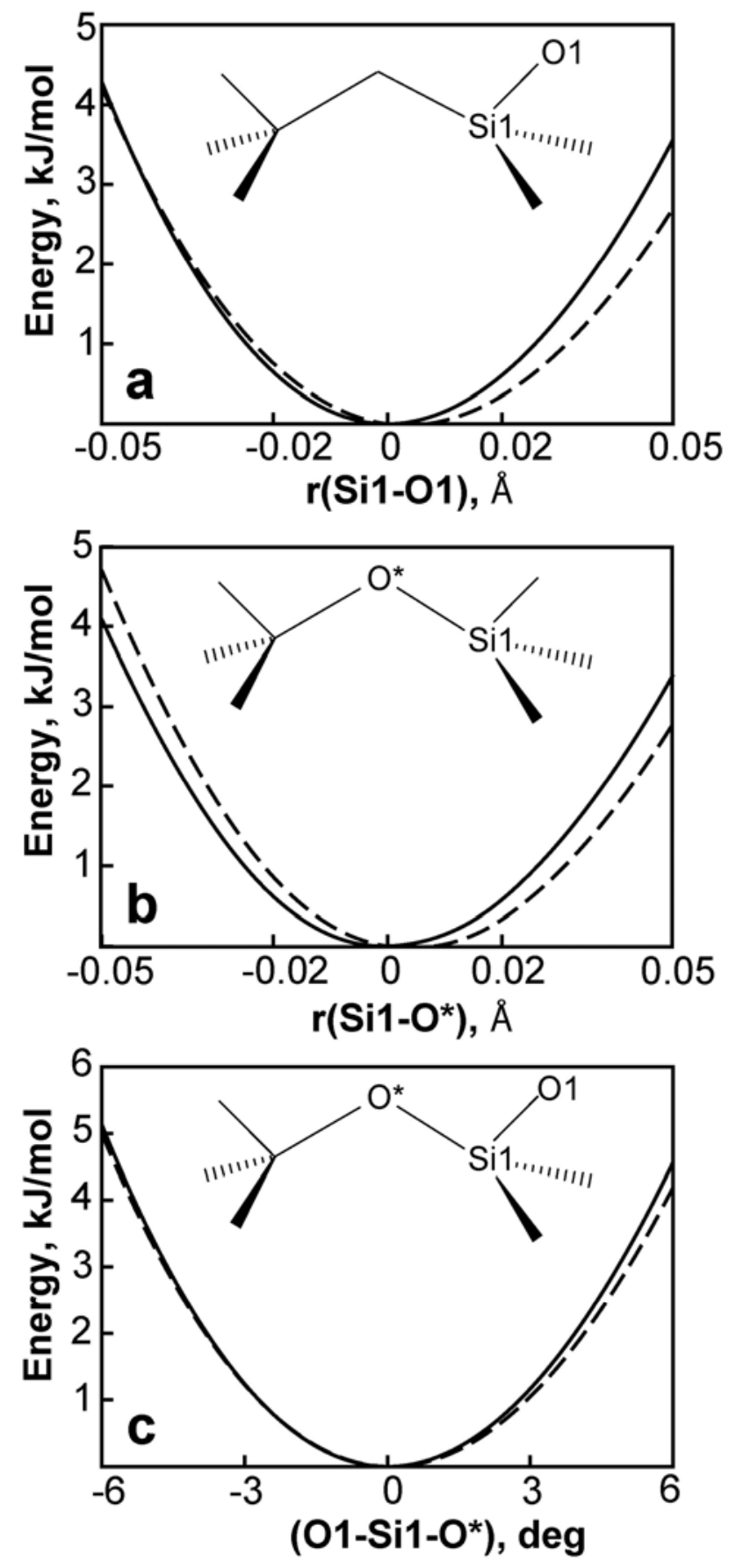

Figure S1. Comparison of various potential energy curves: (a) Si1-O1 bond, (b) Si1-O* bond, and (c) O1-Si1-O* angle in the systems shown in Fig. 2: reference (QM; dashed line) and target (QM/MM; solid line). The curves were obtained by varying (a) the bond lengths Si1-O1, (b) Si1-O* and (c) the bond angle O1-Si1-O*, keeping all other internal coordinates fixed at their values as optimized for the reference system. 